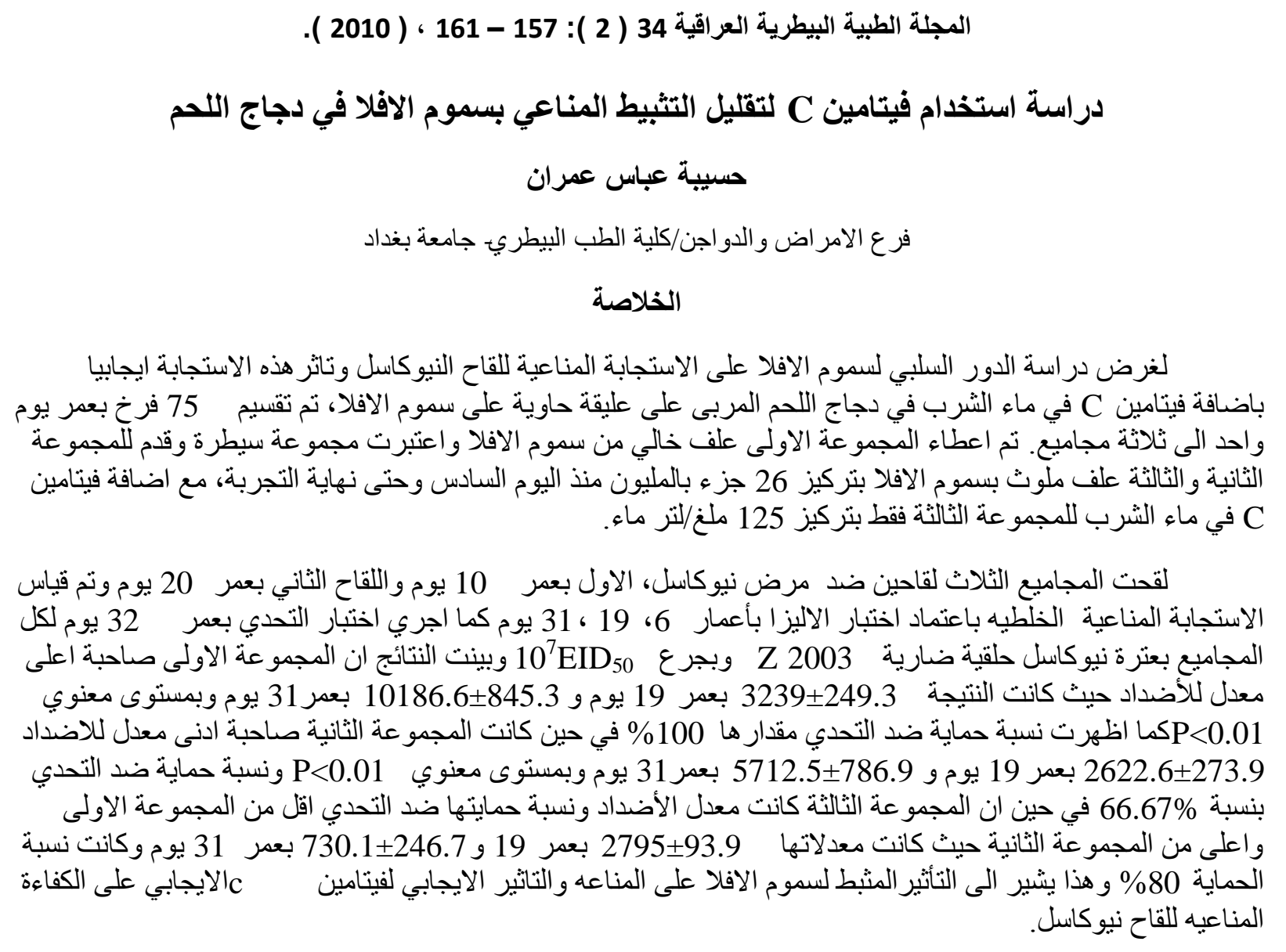

\title{
Study of Using Vit. C to Reduce the Toxic Immunosuppressive Effect of Aflatoxin in Broilers
}

\author{
Hassiba A. Omran \\ Department of poultry disease and pathology- veterinary medicine college-Baghdad University
}

Accepted- $\quad / 2010$.

\section{Summary}

This study was conducted to investigate the adverse effect of aflatoxine contaminated ration on immune response induced by $\mathrm{N} \mathrm{D}$ vaccine in broilen and the effect of adding vit $\mathrm{C}$ in drinking water in reducing the aflatoxine immune supportive effect.

A total of 75 day old broiler chicken were divided randomly in to three equal groups, the first group was feed aflatoxine free ration while second and third groups were feed on aflatoxine contaminated ration at 6 day of age, in addition to that the third group have received vit $\mathrm{C}$ in a drinking water $(125 \mathrm{mg} / \mathrm{litter})$. The birds were immunized twice with ND vaccine at 10, 20 day of age and ELISA test was carried out to estimate the humeral immunity response at $6,19,31$, days of age.

All the groups were challenged with velogenic ND strain (Z 2003). At 32 day of age. The results showed that the first group has high significant antibodies titer reach to $3239 \pm 249.3$ at 19 days of age and $10186 \pm 845.3$ at 31 days of age with significant level $p<0.01$ and high protection ratio against challenge $(100 \%)$ at 32 days of age, while the second group showed a low antibodies titer reach to $2622.6 \pm 27.3$ at the 19 days of age and $5712.5 \pm 786.9$ at 31 days of age and low protection ratio against challenge $(66.67 \%)$. the third group showed a high significant antibodies titer reach to $2795 \pm 930.9$ at 19 days of age and $7030.1 \pm 246.7$ at 31 days 


\section{المجلة الطبية البيطرية العراقية 34 ( 2 ): 157 - 161 ، ( 2010 ).}

of age with significant level $\mathrm{p}<0.01$ with a protection ratio against challenge $80 \%$ at 32 days of age which mean that the third group showed antibodies titer and protection ratio lower than the first group and higher than second group. This study was concluded that giving diets contaminated with aflatoxine cause adverse effects on immune response while the administration of vit $\mathrm{C}$ in drinking water improves it .

\section{المقدمة}

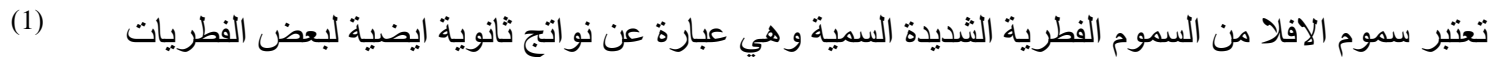

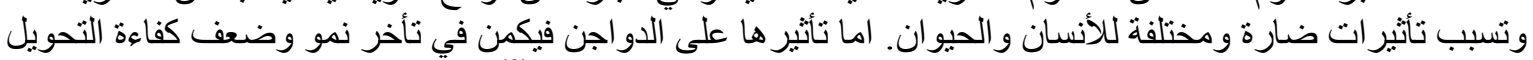

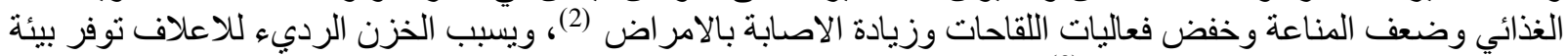

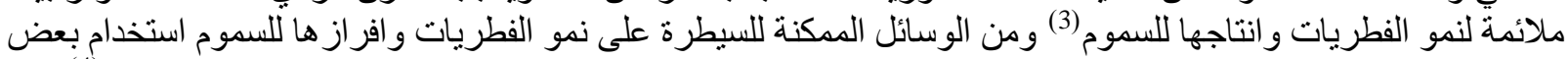

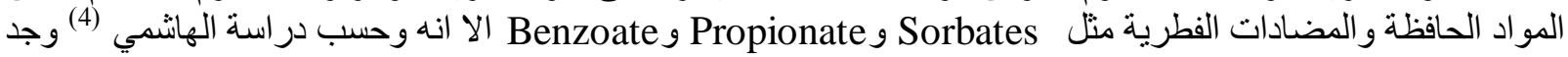
انها تؤثر سلبيا على الاستجابة المناعية للقاح نيوكاسل.

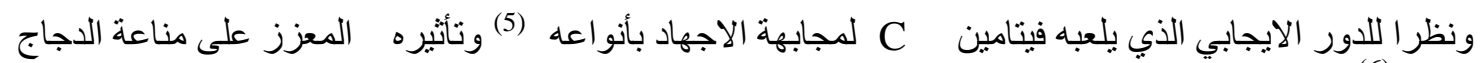

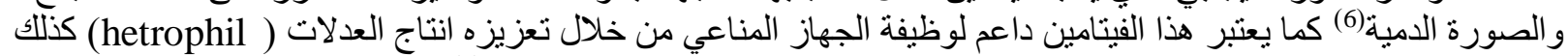

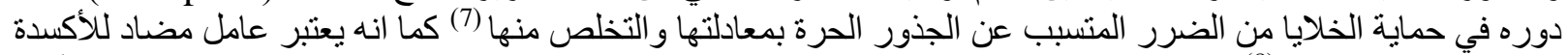

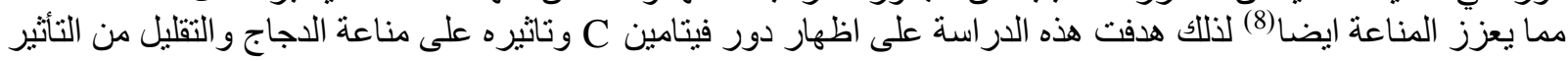

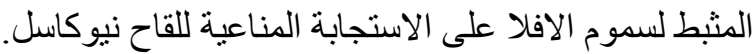

\section{المواد وطرائق العمل}

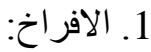

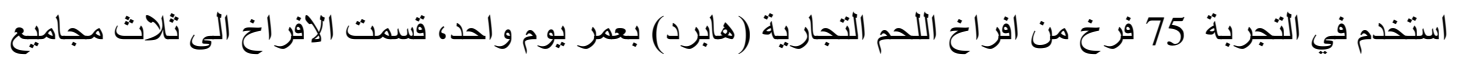

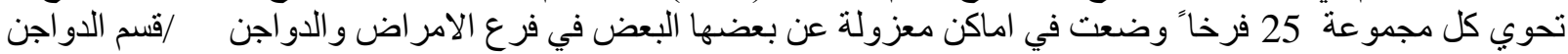
و عرضت الى نفس ظروف عة التربية و الاداره وعوملت أمن كما يلي:

المجمو عة الاولى (م1): اعطيت عليقة خالية من التلوث بسموم الافلا الفطرية.

المجمو عة الثانية (م2): اعطيت ومنذ اليوم السادس من عمر الطير عليقة ملوثة بسموم الاففلا الفطرية وبنركيز 26 جزء من المليون والتي تم قياسه في فرع الصعة الصحة العامه.

المجموعة الثالثة (م3): ): اعطيت ومنذ اليوم السادس من عمر الطير عليقة ملوثة بسموم الافلا الفطرية وبتركيز

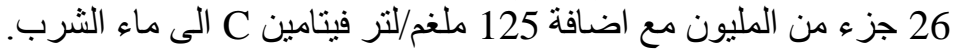

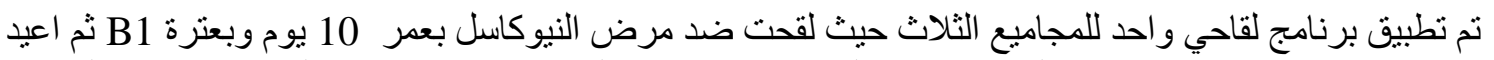

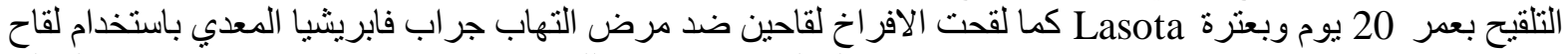

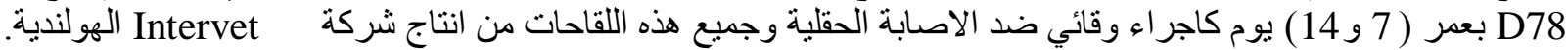

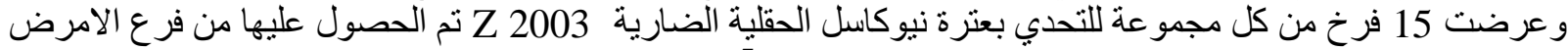

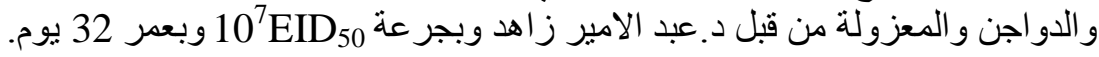

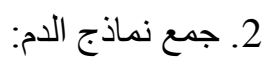

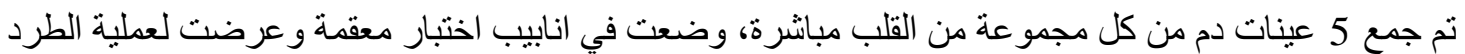

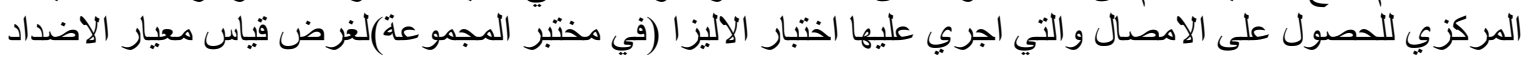

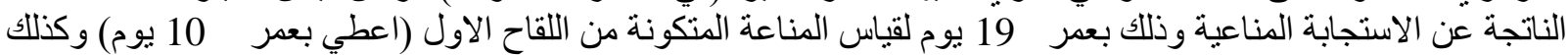

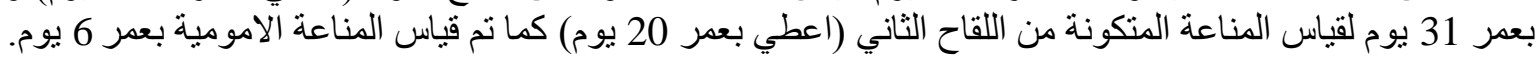

$$
\text { النتائج }
$$

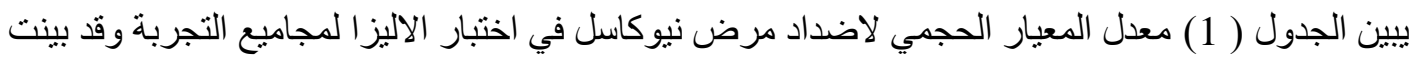

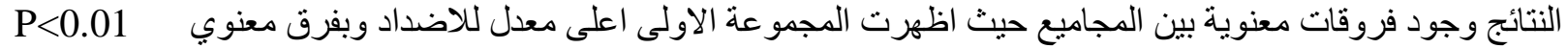
عن المجمو عتين الثانية والثالثة تلتها المجموعة الثالثة و التي كان معدل الاضداد فيها اعلى من المجموعة الثانية وبفرق 


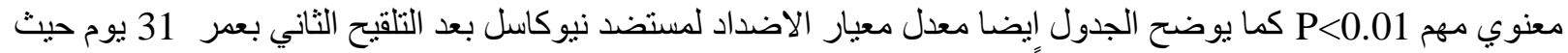

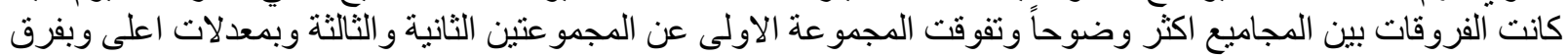

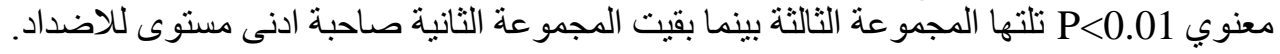

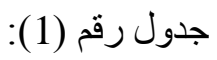

\begin{tabular}{|c|c|c|c|}
\hline $3 p$ & $2 p$ & ק & العمر (يوم) \\
\hline $\begin{array}{c}\mathrm{b} \\
2795 \pm 930.9\end{array}$ & $\begin{array}{c}a \\
2622.6 \pm 373.9\end{array}$ & $\begin{array}{c}c \\
3239.3 \pm 249.3\end{array}$ & 19 \\
\hline $\begin{array}{c}\mathrm{b} \\
7030.1 \pm 246.7\end{array}$ & $\begin{array}{c}\mathrm{a} \\
2712.5 \pm 786.9\end{array}$ & $\begin{array}{c}c \mathrm{c} \\
101186.6 \pm 845.3\end{array}$ & 31 \\
\hline
\end{tabular}

* الحروف المختلفة تمثل وجود فروقات معنوية80.01>01 بين المجاميع بنفس العمر.

* علما ان المناعة الامية لافراخ التجربة كانت2460 (معدل الاضداد).

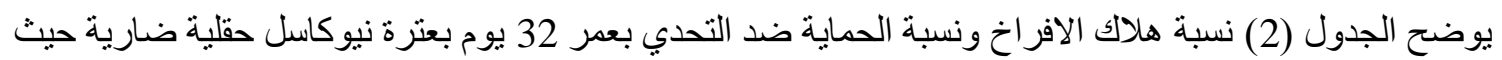

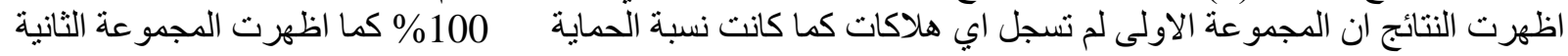

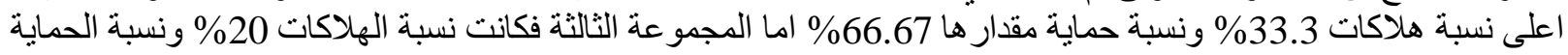

.\%80

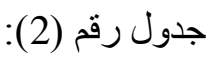

\begin{tabular}{|c|c|c|c|}
\hline نسبة الحماية & نسبة الهلاكات & عدد الههلاكات & المجموعة \\
\hline$\% 100$ & $0 \%$ & $0 / 15$ & ק1 \\
\hline$\% 66.67$ & $33.3 \%$ & $5 / 15$ & 25 \\
\hline$\% 80$ & $20 \%$ & $3 / 15$ & קR \\
\hline
\end{tabular}

المناقشة

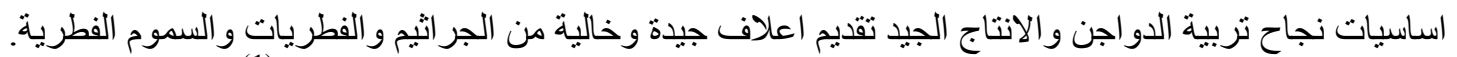

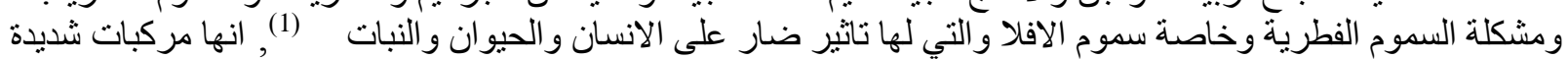

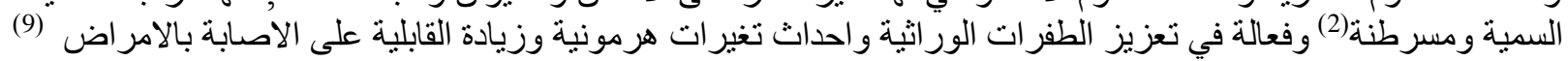

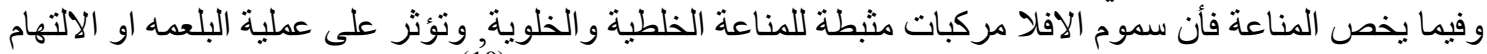

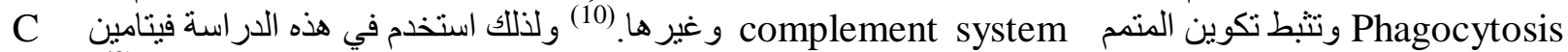

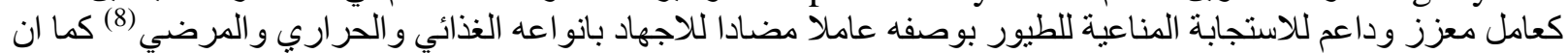

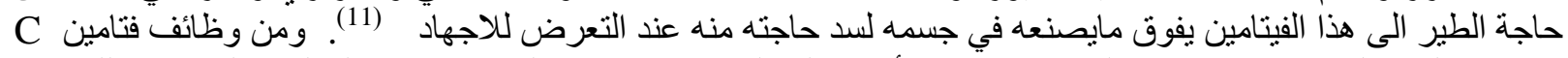

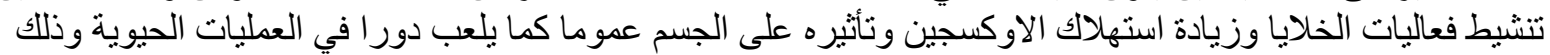

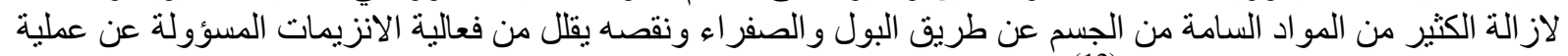

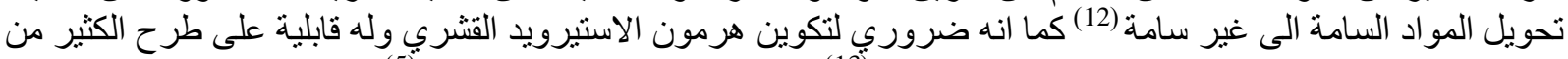

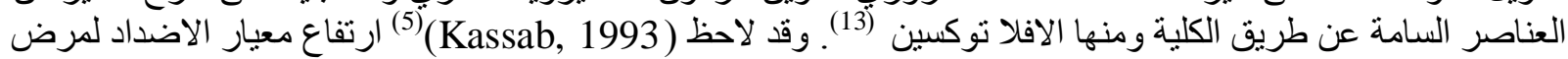

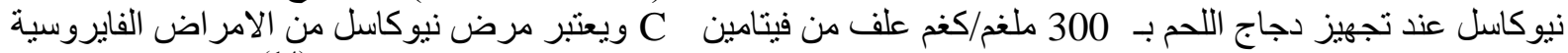

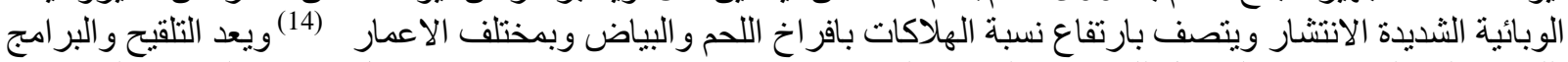

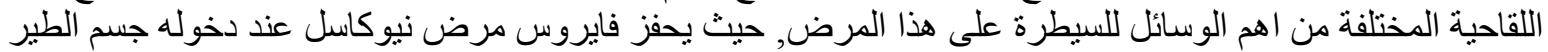

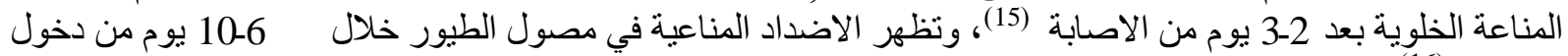

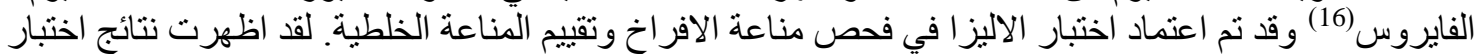

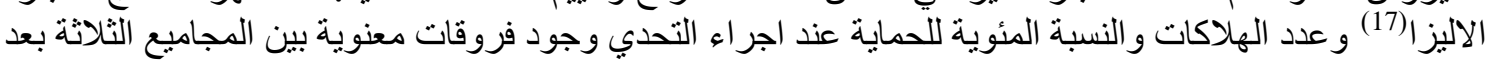

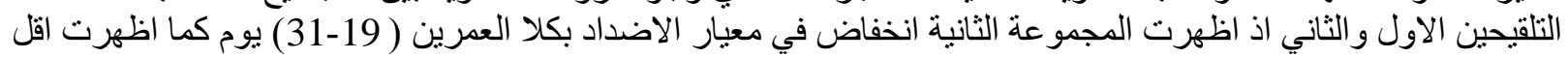

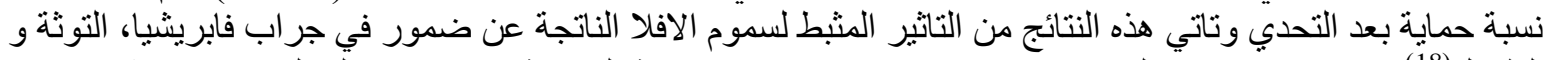

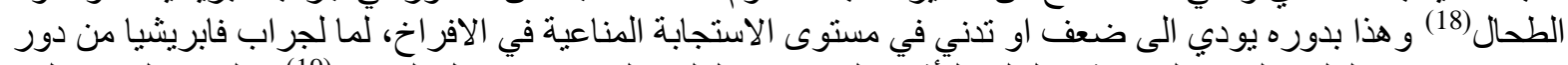

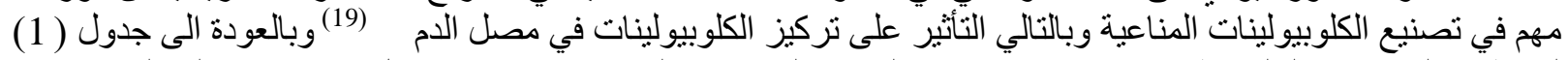

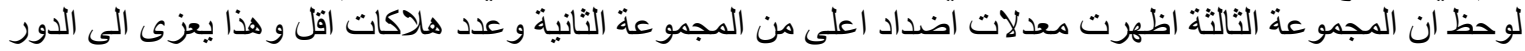
الايجابي الذي يلعبه فيتامين C في تحسين الاستجابة المناعية في الافر اخ. من خلال مساهمته في زيادة انقسام الخلايا اللمفية 
B-cells وتكوين خلايا البلازما وتكوين الاضداد (12)، كما يزيد من عداد وفعالية الخلايا

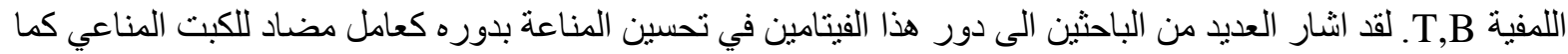

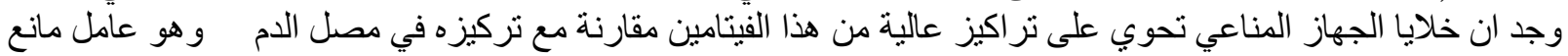

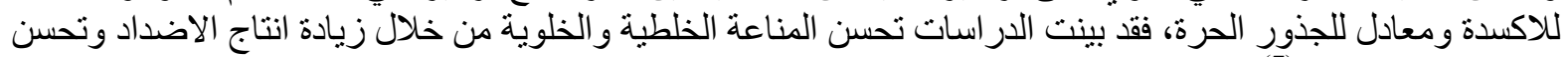

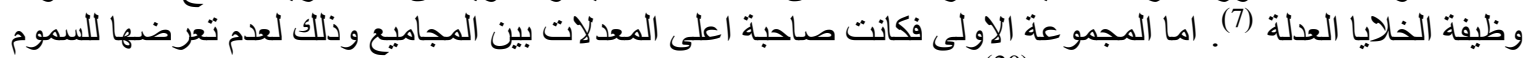
الفطرية التي تعرقل الاستجابة المناعية (20).

\section{المصادر}

1. Pier AC (1973). An over view of the mycotoxicosis of domestic nimals. J Am Vet Med Assoc. 163: 1259-1269.

2. Edds GT (1979). Aflatoxins. In: conference on mycotoxins in animal feeds and related to animal health. Adited by shimoda, w., food and drug administration report no 0 FDA/BVM. 79/139. PP 0 80/164 (cited by calnek, (1997).

3. Edds GT and Bortell RA (1983). Biological effects of alfatoxins in poultry In: alfatoxin and aspergillus flavus in corn. Edited by Diener, U.L., asquith, R.L., and Dickens, J.W., southern Cooperative Series Bulletin 279, Auburn University, Auburn, Al. pp: 56-61.

$$
\begin{aligned}
& \text { 4. الهانثي، لهيب محمد. ( 2000). تاثير استخدامات المضادات الفطرية على المناعة ضد مرض نيوكاسل في دجاج الحم. } \\
& \text { رسالة دبلوم/ كلية الطب البيطري/ جامعة بغداد. }
\end{aligned}
$$

5. Kassab A Al-attar MA and Al-Taweel RN (1993). Effect of ascorbic acid on chickens experimentally infected with Escherishia coli. The veterinarian 3.

6. Mckee JS and Harrison PC (1995). Effect of supplemental ascorbic acid on the performance of broiler chickens exposed to multiple concurrent stressors poult. Sci. 74: 1772-1785.

7. Bendich A (1992). Ascorbic acid \& immune function (Review). Proceeding of the 2nd Symposium, Ascorbic Acid in Domastic animals. Ittingen, Switzerland. Pp: 408-421.

8. Ferkat PR and Quershi MA (1992). Performance and immunity of heat stressed broilers fed vitamin and electrolyte- supplemented drinking water. Poult Sci. 71: 88-97.

9. Hoerr FJ (1997). Poisons and toxins. In: Diseases of poultry 10th ed., Edited by Calnek, B.W. Barnes, H.J.; Beard, C.W.; Mcdougald, L.R. and Salf, Y.M. Mosby- Wolf; USA. PP. 951-978.

$$
\text { 10. نوار، مصطفى ورشاد الناطور ( 1989). الميكونوكسينات و التسمح الميكوتوكسيني في الانسان و الحيوان، الطبعة }
$$

11. Pardue SC and Thaxton JP (1986). Ascorbic acid in poultry. A review. World's Poult. Sci. 42: $107-123$.

12. Klob E (1985). Recent findings on the importance and metabolism of ascorbic acid in domestic animals Mh. Vet. 40: 489-494.

13. Agudelo LG (1983). Possible causes of avian oedema. Poult. Intern. July, 8-14.

14. Alexander DJ (2003). Newcastle disease of poultry. in: "Diseases of poultry", 11th ed. Edited by Y.W. saif, H.J. Barnes, A.M. Fadly, J.R. Glisson, L.R. MCs Douglad and Snayne. Iowa state university press, Ames, Lowa, U.S.A. PP: 63-81. 


\section{المجلة الطبية البيطرية العراقية 34 ( 2 ): 157 - 161 ، ( 2010 ).}

15. Timms L and Alexander DJ (1977). Cell mediated immunoresponse of chickens to Newcastle disease vaccines. Avian Pathol. 6:51-59.

16. Alexander D (1996). Newcastle disease and other paramyxo virus. In: "Diseases of poultry" Eds by. Calek, B.W.; Barnes, H.D.; Beard, C.W.; Mcdoglad, L.R. and Saif, Y. M. 10th ed., Iowa state university press, Ames, Lowa, U.S.A. PP: 541.

17. Synder DB Marquadt WW Mallinson ET Savage PK and Allen DC (1984). Rapid serological profiling by enzyme- linked immunosorbent assay. Avian Dis. 28: 12-24.

18. Cook ME (1996). Diet in duced immunosuppression in: poultry immunology, 1st ed.,P: 318.

19. Glick B (1977). The bursa of fabriius and immunoglobulin synthesis. Int. Rev. Cytol., 48:345-402.

20- Forag RS Daw ZY Hewedi FM and El-Baroty GS (1989). Antimicrobial activity of some Egyptain speceessential Ols.J Food Protection. 52: 665- 667. 